\title{
Temporal Dynamics of ICP, CPP, PRx, and CPPopt in High-Grade Aneurysmal Subarachnoid Hemorrhage and the Relation to Clinical Outcome
}

\author{
Teodor Svedung Wettervik * (i), Timothy Howells, Anders Lewén, Elisabeth Ronne-Engström and Per Enblad
}

\begin{abstract}
Background: High intracranial pressure (ICP) and low cerebral perfusion pressure (CPP) may induce secondary brain injury following aneurysmal subarachnoid hemorrhage (aSAH). In the current study, we aimed to determine the temporal incidence of insults above/below certain ICP/CPP thresholds, the role of pressure autoregulation in CPP management (PRx and CPPopt), and the relation to clinical outcome.

Methods: In this retrospective study, 242 patients were included with aSAH, who were treated in the neurointensive care unit, Uppsala University Hospital, Sweden, 2008-2018, with ICP monitoring the first 10 days post-ictus. Data from ICP, pressure autoregulation (PRx), CPP, and CPPopt (the CPP with the lowest/optimal PRx) were analyzed the first 10 days. The percentage of good monitoring time (GMT) above/below various ICP and CPP thresholds was calculated, e.g., ICP $>20 \mathrm{~mm} \mathrm{Hg}(\%), C P P<60 \mathrm{~mm} \mathrm{Hg}(\%)$, and $\triangle$ CPPopt (CPP-CPPopt) $<-10 \mathrm{~mm} \mathrm{Hg}(\%)$.

Results: Of the 242 patients, 63 (26\%) had favorable (GOS-E 5-8) and 179 (74\%) had unfavorable (GOS-E 1-4) outcome at 12 months. Higher proportion (GMT) of ICP insults above $20 \mathrm{~mm} \mathrm{Hg}$ was most common the first 3 days postictus and was then independently associated with unfavorable outcome. CPP gradually increased throughout the 10 days post-ictus, and higher proportion of GMT with CPP $<90 \mathrm{~mm} \mathrm{Hg}$ was independently associated with unfavorable outcome in the late vasospasm phase (days 6.5-10). PRx was above 0 throughout the 10 days and deteriorated in the late vasospasm phase. Higher values were then independently associated with unfavorable outcome. There was no difference in GMT of CPP deviations from CPPopt between the outcome groups.
\end{abstract}

Conclusions: Avoiding intracranial hypertension early and maintaining a high CPP in the vasospasm phase when the pressure autoregulation is most disturbed may improve clinical outcome after aSAH.

Keywords: Cerebral perfusion pressure, Clinical outcome, Neurointensive care, Pressure autoregulation, Secondary insults, Subarachnoid hemorrhage

\section{Introduction}

Aneurysmal subarachnoid hemorrhage (aSAH) constitutes $5 \%$ of all cases of stroke and is associated with high mortality and severe neurological sequelae [1]. Clinical

*Correspondence: teodor.svedung-wettervik@neuro.uu.se Department of Neuroscience, Section of Neurosurgery, Uppsala University, 75185 Uppsala, Sweden management aims at early aneurysm occlusion, cerebrospinal fluid (CSF) diversion in case of acute hydrocephalus, and avoiding delayed cerebral ischemia (DCI) [2-5]. Treatment in the neurointensive care (NIC) has generally led to better clinical outcome for these patients $[3,4,6]$. In an early NIC study, we found that a higher incidence of secondary insults in general was associated with unfavorable outcome after aSAH [3]. Specifically, secondary 
intracranial pressure (ICP) insults above 20 and $25 \mathrm{~mm}$ $\mathrm{Hg}$ are relatively common post-ictus [4, 7-9] and associated with clinical deterioration [4] and mortality [7], but the relation to favorable/unfavorable clinical outcome is less clear $[4,7,8]$. Regarding cerebral perfusion pressure (CPP; mean arterial blood pressure-ICP) insults, the evidence for the benefit of fixed CPP thresholds is low. CPPs above $60-70 \mathrm{~mm} \mathrm{Hg}$ are generally desired $[4,10]$ and levels below $70 \mathrm{~mm} \mathrm{Hg}$ have been suggested to be associated with brain tissue hypoxia and energy metabolic disturbances due to impaired cerebral blood flow (CBF) [10]. However, aSAH has a dynamic CBF pathophysiology with cerebral large-vessel vasospasm that peaks days 4 to 10 post-ictus and that contributes to the development of DCI $[5,11]$. The implication of this is that the goal for CPP treatment could vary.

One way of monitoring the changes in the CBF pathophysiology is continuous assessment of the pressure autoregulatory status, which could ultimately lead to a more individualized and dynamic CPP management. In TBI, pressure autoregulation has been monitored using the pressure reactivity index (PRx), i.e., a correlation index between ICP and mean arterial blood pressure (MAP). Negative values, e.g., when a decrease in MAP leads to cerebral vasodilation to maintain $\mathrm{CBF}$ with a corresponding increase in cerebral blood volume and ICP, indicate preserved pressure autoregulation and are strongly associated with favorable outcome in TBI [12, 13]. As PRx varies with CPP in a U-shaped way, the CPP with the concurrently lowest $\mathrm{PRx}$ /best autoregulation has been suggested as optimal (CPPopt). Deviation of absolute CPP from CPPopt has been associated with poor clinical outcome in TBI [13-15]. Some studies suggest that disturbed PRx values and deviation from CPPopt targets are associated with low CBF in aSAH $[16,17]$, but their ability to predict DCI and clinical outcome are less clear [16-22].

Although ICP/CPP insults and disturbances in cerebral autoregulation are common following aSAH, their temporal evolution and relation to clinical outcome are not fully elucidated. In the current study, our aim was to evaluate the temporal incidence of ICP and CPP insults based on different threshold values and the role of pressure autoregulation in relation to clinical outcome. We were particularly interested in evaluating fixed CPP thresholds as compared to the autoregulatory CPPopt thresholds in relation to clinical outcome.

\section{Materials and Methods Patients}

Patients with aSAH admitted to the Department of Neurosurgery at the University Hospital in Uppsala, Sweden, 2008-2018 were eligible for this study. Out of 605 patients with SAH and ICP monitoring, we included 242 aSAH patients aged 16 or older with ICP/CPP monitoring on all of the first 10 days post-ictus.

\section{Treatment Protocol}

Patients were treated in accordance with our standardized ICP- and CPP-oriented treatment protocol to avoid secondary insults [4]. The treatment protocol remained unchanged throughout the study period. Treatment goals were $\mathrm{ICP} \leq 20 \mathrm{~mm} \mathrm{Hg}, \mathrm{CPP} \geq 60 \mathrm{~mm} \mathrm{Hg}$, systolic blood pressure $>100 \mathrm{~mm} \mathrm{Hg}$, central venous pressure (CVP) 0-5 $\mathrm{mm} \mathrm{Hg}$ before the aneurysm was occluded and 5-10 mm Hg afterward, $\mathrm{pO}_{2}>12 \mathrm{kPa}$, arterial glucose $5-10 \mathrm{mmol} / \mathrm{L}(\mathrm{mM})$, electrolytes within normal ranges, normovolemia and body temperature $<38^{\circ} \mathrm{C}$.

Patients who were unconscious $(\mathrm{GCS} M<6)$ were intubated and mechanically normoventilated. Those patients were sedated with propofol and received morphine as analgesia. Wake-up tests were repeatedly performed. The patients were treated with early aneurysm occlusion, including endovascular embolization or surgical clipping, and all patients received nimodipine (first as infusion of $2 \mathrm{mg} / \mathrm{h}$ and later as tablets $60 \mathrm{mg} \times 6$ for 3 weeks in total). The dosage of infusion was reduced temporarily in case of hypotension to avoid negative hemodynamic effects. In unconscious $(\mathrm{GCS} M<6)$ patients, an external ventricular drain (EVD) was inserted to monitor and to drain cerebrospinal fluid (CSF) in case of high ICP. The EVD was initially kept closed to measure ICP and assess the need for CSF drainage. If ICP was above $20 \mathrm{~mm} \mathrm{Hg}$ the EVD was opened at $15 \mathrm{~mm} \mathrm{Hg}$. In severe cases when basal ICP treatment was insufficient, thiopental coma treatment and/or decompressive craniectomy (DC) were last-tier treatments. Arterial blood pressure was maintained with fluids. Inotropes (dobutamine or in second hand norepinephrine) were only used if CPP was below $60 \mathrm{~mm} \mathrm{Hg}$ and the patient did not respond to intravenous fluid treatment.

DCI was defined as new neurological deficits and/or decreased level of consciousness when other causes, e.g., hydrocephalus and hematomas, were excluded. If a manifest cerebral infarction was excluded, triple- $\mathrm{H}$ therapy (hypertension, hypervolemia, and hemodilution) including $500 \mathrm{ml}$ dextran- 40 solution $(100 \mathrm{mg} / \mathrm{ml}$, Meda AB) and $200 \mathrm{ml}$ albumin $(200 \mathrm{mg} / \mathrm{ml})$ were administered for 5 days. Cerebral intra-arterial nimodipine was given in case of refractory vasospasm and angioplasty was performed in case of refractory large-vessel vasospasm. The main target for triple-H therapy was elevation of blood pressure but only to moderately elevated levels in relation to baseline, i.e., in general CPP to around $70-80 \mathrm{~mm}$ $\mathrm{Hg}$ and systolic blood pressure to around 140-160 mm $\mathrm{Hg}$. Secondary targets were erythrocyte volume fraction 
(EVF) 32\% and CVP 8-14 mm Hg, although these goals were in general met automatically by the fluid therapy given. The targeted levels were increased stepwise if clinical improvement was not seen.

\section{Data Acquisition and Analyses}

The data were collected using our software Odin, developed at Edinburgh and Uppsala University by one of the authors (TH) [23]. ICP was monitored with the EVD system (HanniSet, Xtrans, Smith Medical GmbH, Grasbrunn, Germany). Arterial blood pressure was measured invasively in the radial artery at heart level. The sampling frequency was $100 \mathrm{~Hz}$. Pressure reactivity index (PRx) was calculated as the 5-min correlation of $10 \mathrm{~s}$ averages of ICP and MAP [12, 13]. CPPopt was calculated continuously, minute-by-minute as the CPP with the lowest PRx the last $4 \mathrm{~h}$, as described by Aries et al. [14]. Daily CPPopt-values could be calculated in 90-99\% of the patients, depending on the day. However, CPPopt could only be calculated for $54 \%$ of the GMT for the entire cohort the first 10 days. The data acquisition was on some occasions interrupted when the patients left the NIC (e.g., for surgery) or due to technical aspects (e.g., network failure). These interruptions and data that were judged invalid were subtracted from the total monitoring time, resulting in the good monitoring time (GMT).

\section{Outcome}

Clinical outcome was assessed at around 12 months posthemorrhage, by specially trained personnel using structured telephone interviews for the Extended Glasgow Outcome Scale (GOS-E) containing eight categories of outcome, from death to upper good recovery [24, 25]. GOS-E 5-8 was considered favorable clinical outcome, whereas GOS-E 1-4 was considered unfavorable.

\section{Statistical Analysis}

The temporal dynamics of the following physiological variables were studied in relation to clinical outcome after aSAH: MAP, ICP, CPP, CPPopt, PRx, and body temperature.

Nominal, ordinal, and continuous variables were described as numbers or proportions, medians (interquartile range (IQR)) and means ( \pm standard deviation), respectively. Mean daily values for MAP, ICP, CPP, CPPopt, PRx, and body temperature were evaluated dayto-day the first 10 days post-ictus for those with favorable/unfavorable outcome and those with triple-H/no triple-H with $95 \%$ confidence interval (CI).

The 10-day period post-ictus was divided into three phases-1. Early phase (days 1 to 3 ), 2 . Early vasospasm phase (days 4 to 6.5 ), and 3. Late vasospasm phase (days 6.5 to 10). The vasospasm phase was hence split in the middle at 6.5 days. The proportion (\%) of GMT with secondary insults of ICP, CPP, and body temperature above/ below certain predefined thresholds were calculated for each phase. We defined ICP insults as ICP above threshold values at $20 \mathrm{~mm} \mathrm{Hg}$ and $25 \mathrm{~mm} \mathrm{Hg}$, respectively. The former was chosen in accordance with our management protocol and the latter as a "severe" insult. We studied GMT (\%) of CPP below threshold values at $60 \mathrm{~mm}$ $\mathrm{Hg}, 70 \mathrm{~mm} \mathrm{Hg}, 80 \mathrm{~mm} \mathrm{Hg}$, and $90 \mathrm{~mm} \mathrm{Hg}$. We chose $60 \mathrm{~mm} \mathrm{Hg}$ in accordance with our management protocol and then increased the threshold with $10 \mathrm{~mm} \mathrm{Hg}$ up to $90 \mathrm{~mm} \mathrm{Hg}$. We defined autoregulatory CPP thresholds as optimal when the absolute CPP was within $\pm 10 \mathrm{~mm} \mathrm{Hg}$ from CPPopt $(\triangle \mathrm{CPPopt}=\mathrm{CPP}-\mathrm{CPPopt} \pm 10 \mathrm{~mm} \mathrm{Hg})$, whereas $\Delta$ CPPopt $<-10 \mathrm{~mm} \mathrm{Hg}$ and $\Delta$ CPPopt $>10 \mathrm{~mm}$ $\mathrm{Hg}$ were considered as hypo- and hyperperfusion insults. We defined hyperthermia insults as body temperature above $38{ }^{\circ} \mathrm{C}$ in accordance with our management protocol. There is no gold standard PRx threshold of autoregulation. We therefore focused on mean values, but evaluated also PRx $>0.05$ which has been used to dichotomize TBI patients in favorable/unfavorable outcome [26]. We compared the incidence of ICP, CPP, hyperthermia, and PRx insults and in each of three phases for those with favorable and unfavorable outcome. Those secondary insults that were significant in the univariate analyses in each phase were analyzed in a multiple logistic regression in addition to age, World federation of neurosurgical society (WFNS) grade, and Fisher grade as explanatory variables for favorable outcome. If, e.g., both ICP thresholds (20 and $25 \mathrm{~mm} \mathrm{Hg}$ ) were significant in the univariate analysis in one phase, each threshold was analyzed in the multiple regression separately and the regression with the highest area under receiver operating curve (AUROC) was chosen as optimal. A $p$ value $<0.05$ was considered statistically significant. As this was an exploratory study, we did not adjust for multiple comparisons.

\section{Ethics}

All procedures performed in the studies involving humans were in accordance with the ethical standards of the national research committee and with the $1964 \mathrm{Hel}-$ sinki declaration and its later amendments. The study was approved by Uppsala University Regional Ethical Board (Dnr 2010/138 and Dnr 2010/138/1). Informed consent was obtained during neurointensive care from the next of kin.

\section{Results}

Demography, Admission Status and Treatments, and Relation to Clinical Outcome

There were 242 patients included in the study (Table 1 ). Mean age was $58( \pm 11)$ and the female/male ratio was 
Table 1 Demography, admission status, and treatments-relation to clinical outcome

\begin{tabular}{lllll} 
& All & Favorable & Unfavorable & $\boldsymbol{p}$ Value \\
\hline Patients, $n(\%)$ & $242(100 \%)$ & $63(26 \%)$ & $179(74 \%)$ & NA \\
Age, mean ( \pm SD) years & $58( \pm 11)$ & $53( \pm 11)$ & $60( \pm 10)$ & $\mathbf{0 . 0 0 1}$ \\
Sex (female/male), $n(\%)$ & $163 / 79(67 / 33 \%)$ & $43 / 20(68 / 32 \%)$ & $120 / 59(67 / 33 \%)$ & 0.86 \\
GCS M, median (IQR) & $5(5-6)$ & $6(5-6)$ & $5(4-6)$ & $\mathbf{0 . 0 0 1}$ \\
Pupillary abnormality, $n(\%)$ & $13(5 \%)$ & $0(0 \%)$ & $13(7 \%)$ & $\mathbf{0 . 0 3}$ \\
WFNS grade (IV-V/III), $n$ (\%) & $183 / 59(76 / 24 \%)$ & $39 / 24(62 / 38 \%)$ & $144 / 35(80 / 20 \%)$ & $\mathbf{0 . 0 0 3}$ \\
Hunt and Hess grade (IV-V/IIII), $n(\%)$ & $110 / 132(55 / 45 \%)$ & $18 / 45(29 / 71 \%)$ & $92 / 87(51 / 49 \%)$ & $\mathbf{0 . 0 0 2}$ \\
Fisher grade, median (IQR) & $4(3-4)$ & $3(3-4)$ & $4(3-4)$ & $149 / 30(83 / 17 \%)$ \\
Aneurysm, anterior/posterior circulation, $n(\%)$ & $196 / 46(81 / 19 \%)$ & $47 / 16(75 / 25 \%)$ & 0.002 \\
Aneurysm treatment, no/endovascular/surgery/both, $n(\%)$ & $4 / 169 / 67 / 2(2 / 70 / 28 / 1 \%)$ & $0 / 50 / 11 / 2(0 / 79 / 18 / 3 \%)$ & $4 / 119 / 56 / 0(2 / 66 / 31 / 0 \%)$ & $\mathbf{0 . 0 1}$ \\
Thiopental, $n$ (\%) & $24(10 \%)$ & $2(3 \%)$ & $22(12 \%)$ & $\mathbf{0 . 0 4}$ \\
Decompressive craniectomy, $n(\%)$ & $34(14 \%)$ & $5(8 \%)$ & $29(16 \%)$ & 0.10 \\
\hline
\end{tabular}

$p$ Values in bold italics are considered statistically significant

163/79 (67/33\%). Median GCS M was 5 (IQR 5-6) and pupillary abnormalities (anisocoria/fixed) were present in $13(5 \%)$ patients at admission. There were $183(76 \%)$ patients with a WFNS grade above III and 110 (55\%) patients with a Hunt and Hess grade above III. Median Fisher grade was 4 (IQR 3-4) and the aneurysm was located in the anterior cerebral circulation for 196 (81\%) patients and in the posterior circulation for the remaining patients. The majority $(\mathrm{n}=169(70 \%))$ of the patients were treated with endovascular aneurysm occlusion, whereas 67 (28\%) were treated with clip ligation, 2 (1\%) patients with both methods, and 4 (2\%) patients received no aneurysm occlusion. All patients were intubated and they were mechanically ventilated in median 12 (IQR 9-15) days. Sixty-one (25\%) patients were treated with triple-H therapy due to DCI, of whom 6 also received cerebral intra-arterial nimodipine and 1 received additional angioplasty. Twenty-four (10\%) patients were treated with thiopental and 34 (14\%) with DC. Ten (4\%) patients were treated with both thiopental and DC.

Of the 242 patients, 63 (26\%) had favorable and 179 (74\%) unfavorable clinical outcome (Table 1). Those with favorable outcome were significantly younger, in a better neurological condition at admission, had less SAH on the first CT scan and were more often treated with endovascular aneurysm occlusion.

Temporal Dynamics of Systemic and Cerebral Physiological Variables, and Relation to Triple-H Treatment and Clinical Outcome

Mean daily values of systemic and cerebral physiological variables the first 10 days post-hemorrhage for all patients are demonstrated in Figs. 1 and 2. The mean daily MAP at admission was around $90 \mathrm{~mm} \mathrm{Hg}$, gradually increased throughout the temporal course to $100 \mathrm{~mm}$
$\mathrm{Hg}$, and was significantly higher for those with favorable outcome in the late vasospasm phase. Those who were treated with triple- $\mathrm{H}$ had higher MAP in the early and late vasospasm phases. Mean ICP remained relatively stable around $10 \mathrm{~mm} \mathrm{Hg}$ throughout the temporal course for both outcome groups. The temporal CPP trend was similar to the MAP curve, with a mean daily CPP that was slightly lower at admission (below $80 \mathrm{~mm}$ $\mathrm{Hg}$ ), gradually increased along the temporal course and was significantly higher for those with favorable outcome in the early and late vasospasm phases. Similarly, those treated with triple- $\mathrm{H}$ had higher $\mathrm{CPP}$ in the same phases. The CPPopt trend showed a similar pattern as for mean CPP and CPPopt was significantly higher in the early $(83 \pm 10 \mathrm{~mm} \mathrm{Hg}$ vs. $81 \pm 8 \mathrm{~mm} \mathrm{Hg}, p$ value $<0.05)$ and late ( $87 \pm 9 \mathrm{~mm} \mathrm{Hg}$ vs. $83 \pm 8 \mathrm{~mm} \mathrm{Hg}, p$ value $<0.01$ ) vasospasm phases for those with favorable outcome. PRx was generally disturbed with mean values above 0 throughout the temporal course for both outcome groups. Those with unfavorable outcome had significantly higher PRx in the late vasospasm period than those with favorable outcome $(0.22 \pm 0.16$ vs. $0.14 \pm 0.12, p$ value $<0.001)$. Those with triple-H treatment did not have a significantly higher PRx. Body temperature increased in both outcome groups from approximately $37^{\circ} \mathrm{C}$ at admission to slightly above $38^{\circ} \mathrm{C}$ in the early and late vasospasm phases.

\section{Temporal Occurrence of Predefined Secondary Insults and Relation to Clinical Outcome}

The incidence of ICP insults (GMT (\%) ICP $>20 \mathrm{~mm} \mathrm{Hg}$ and GMT (\%) > $25 \mathrm{~mm} \mathrm{Hg}$, respectively) were higher in the early phase post-ictus (Fig. 3 and Table 2). Patient with favorable outcome had significantly lower GMT (\%) of ICP above both thresholds in the early phase (above $20 \mathrm{~mm} \mathrm{Hg}, 3 \pm 3$ vs. $6 \pm 9, p$ value $<0.001$ and above 
MAP (mm Hg)

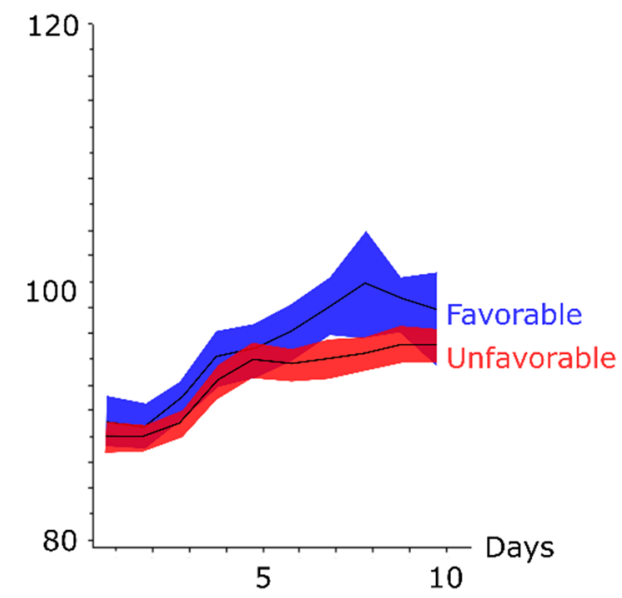

CPP (mm Hg)

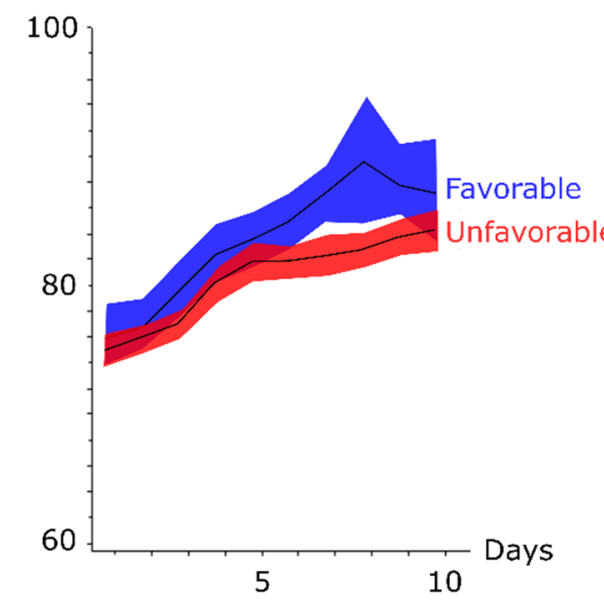

PRx (coefficient)

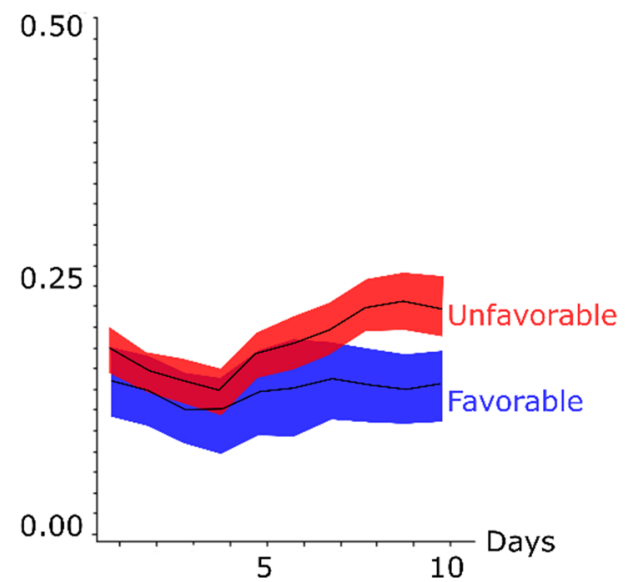

ICP $(\mathrm{mm} \mathrm{Hg})$

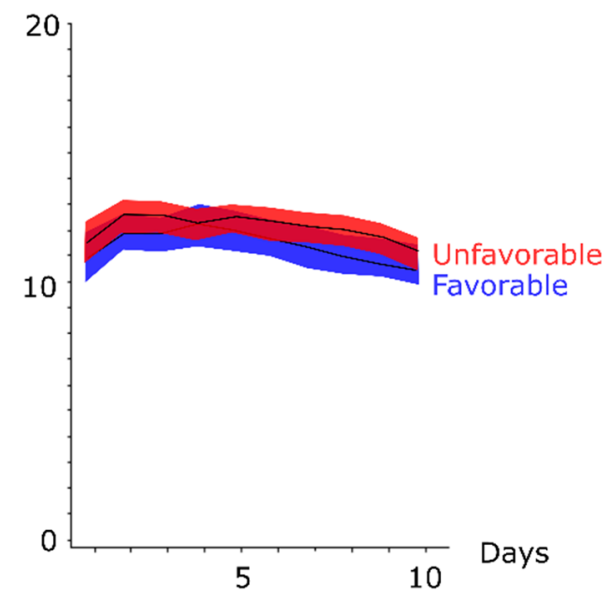

CPPopt (mm Hg)

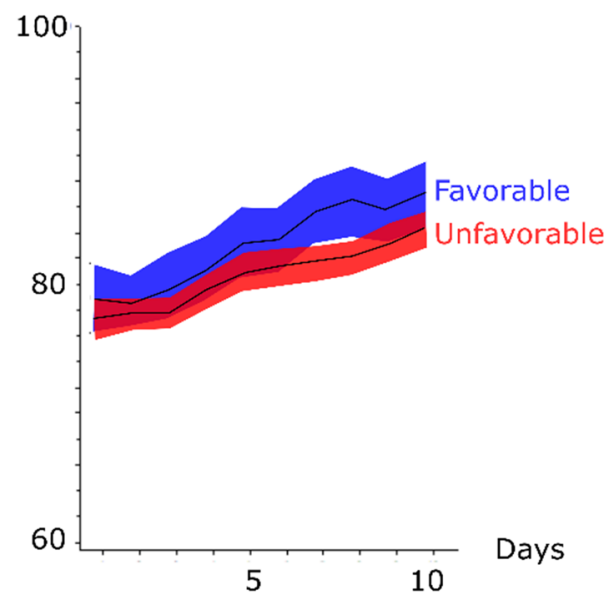

Temperature (C)

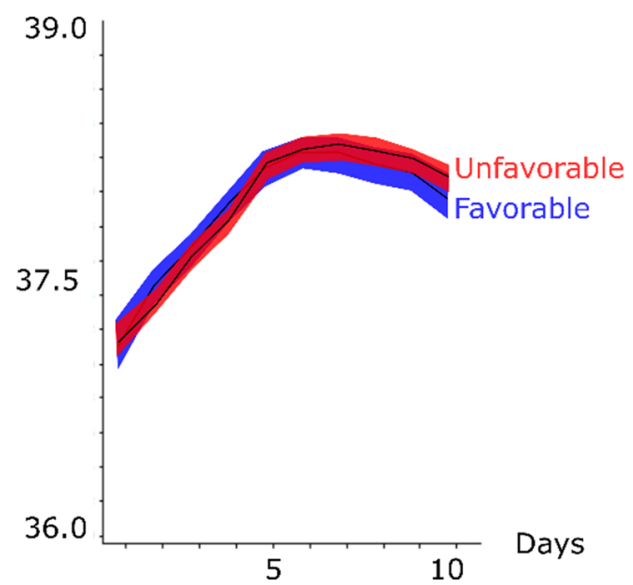

Fig. 1 Systemic and cerebral physiological variables in relation to clinical outcome following aneurysmal subarachnoid hemorrhage-a temporal analysis the first 10 days. The trendlines indicate mean values and the shaded areas (red and blue) the $95 \%$ confidence interval. CPP $=$ Cerebral perfusion pressure, CPPopt $=$ optimal CPP (the CPP with the concurrently best autoregulation), ICP $=$ Intracranial pressure, MAP $=$ Mean arterial blood pressure, and $\mathrm{PRx}=$ Pressure reactivity index 
MAP (mm Hg)

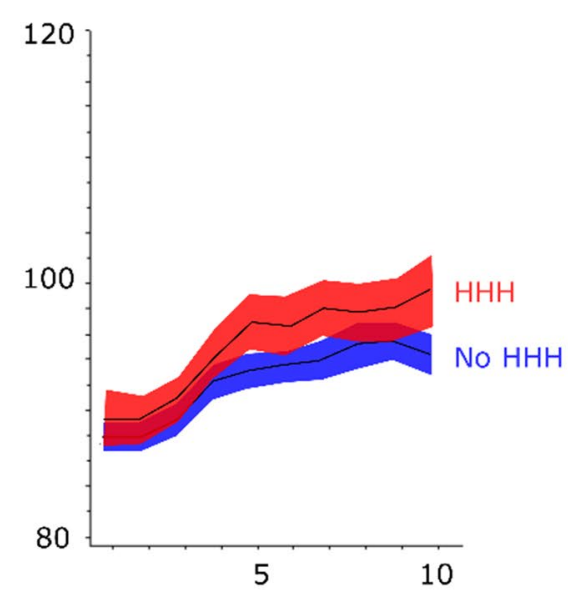

CPP $(\mathrm{mm} \mathrm{Hg})$

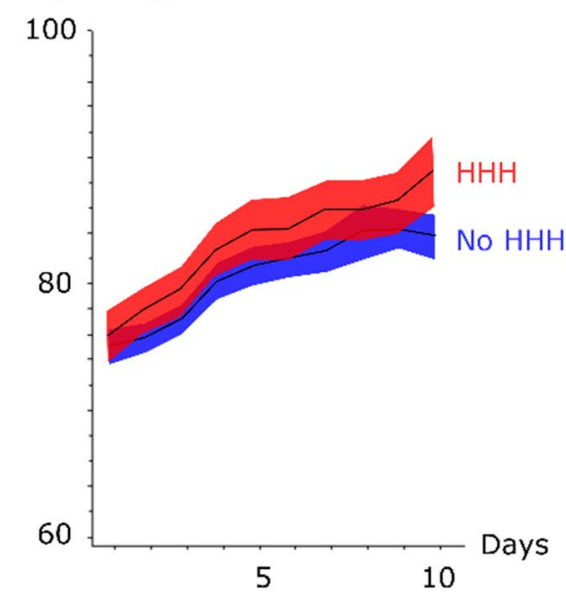

PRx (coefficient)

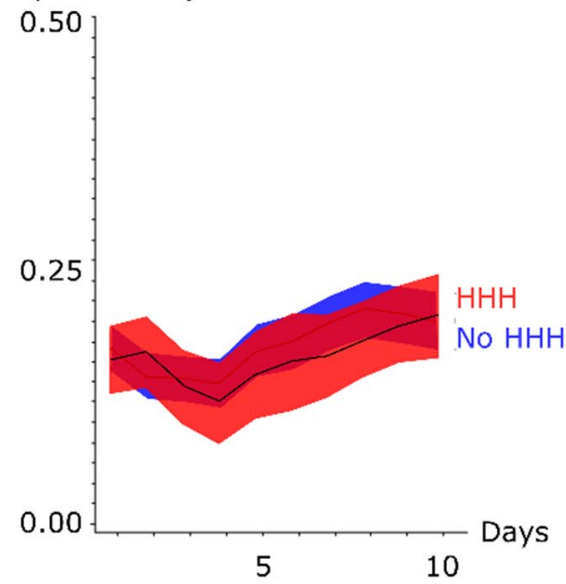

ICP $(\mathrm{mm} \mathrm{Hg})$

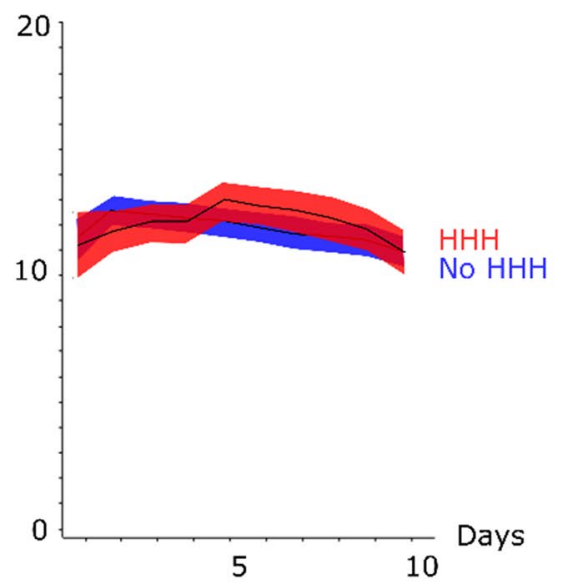

CPPopt (mm Hg)
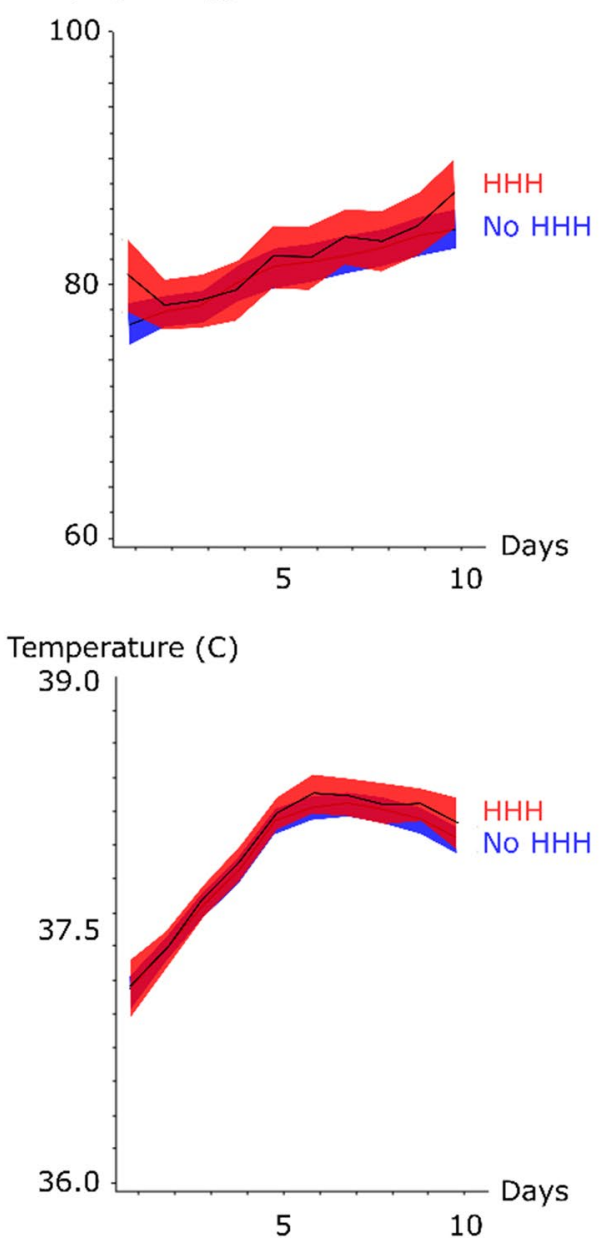

Fig. 2 Systemic and cerebral physiological variables in relation to triple-H therapy following aneurysmal subarachnoid hemorrhage-a temporal analysis the first 10 days. The trendlines indicate mean values and the shaded areas (red and blue) the $95 \%$ confidence interval. CPP $=$ Cerebral perfusion pressure, CPPopt $=$ optimal CPP (the CPP with the concurrently best autoregulation), ICP $=$ Intracranial pressure, MAP $=$ Mean arterial blood pressure, and $\mathrm{PRx}=$ Pressure reactivity index 
ICP $>20 \mathrm{~mm} \mathrm{Hg}(\% \mathrm{GMT})$

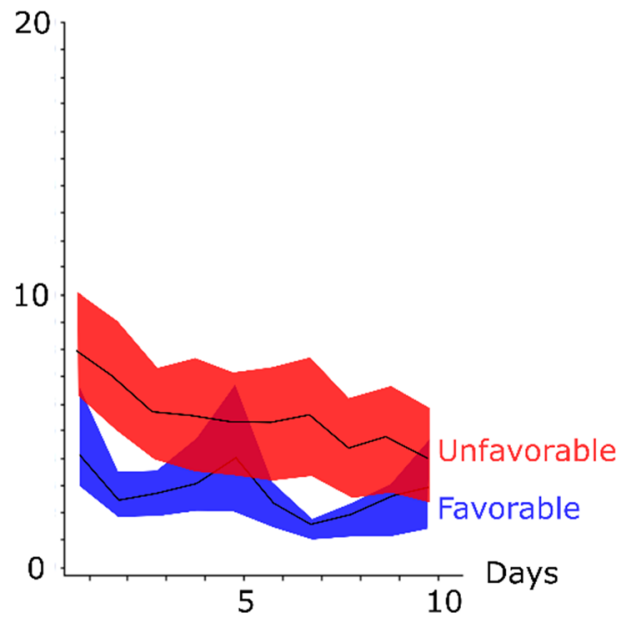

ICP $>25 \mathrm{~mm} \mathrm{Hg}(\% \mathrm{GMT})$

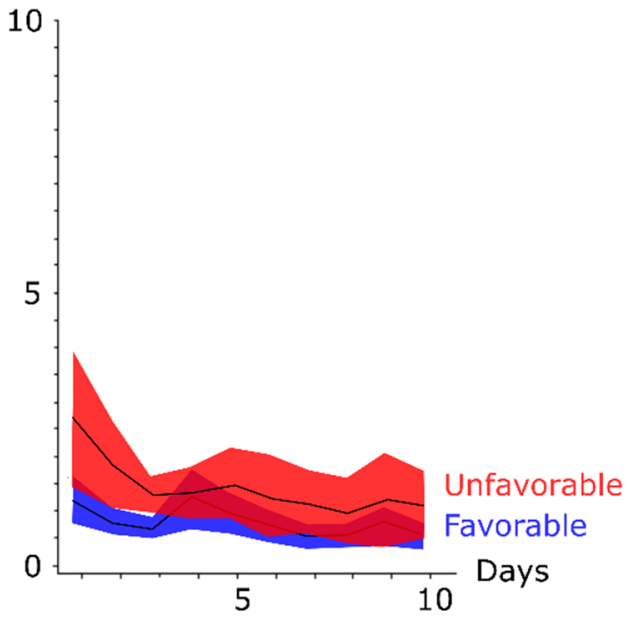

Fig. 3 Intracranial pressure thresholds in relation to clinical outcome following aneurysmal subarachnoid hemorrhage-a temporal analysis the first 10 days. The trendlines indicate mean values and the shaded areas (red and blue) the 95\% confidence interval. GMT=Good monitoring time and ICP $=$ Intracranial pressure

Table 2 Secondary insults in relation to clinical outcome following aneurysmal subarachnoid hemorrhage-a temporal analysis of three phases the first 10 days

\begin{tabular}{|c|c|c|c|c|c|c|c|c|c|}
\hline \multirow[t]{2}{*}{ Variables } & \multicolumn{3}{|l|}{ Ictal phase } & \multicolumn{3}{|c|}{ Early vasospasm phase } & \multicolumn{3}{|c|}{ Late vasospasm phase } \\
\hline & Favorable & Unfavorable & $p$ Value & Favorable & Unfavorable & $p$ Value & Favorable & Unfavorable & $p$ Value \\
\hline ICP > 20 mm Hg (\%) & $3( \pm 3)$ & $6( \pm 9)$ & 0.001 & $3( \pm 4)$ & $5( \pm 13)$ & 0.04 & $2( \pm 3)$ & $4( \pm 12)$ & 0.01 \\
\hline $\mathrm{ICP}>25$ mm Hg (\%) & $1( \pm 1)$ & $2( \pm 2)$ & 0.001 & $1( \pm 1)$ & $1( \pm 3)$ & 0.30 & $1( \pm 1)$ & $1( \pm 4)$ & 0.35 \\
\hline CPP < 60 mm Hg (\%) & $4( \pm 5)$ & $6( \pm 7)$ & 0.09 & $2( \pm 3)$ & $3( \pm 4)$ & 0.01 & $1( \pm 2)$ & $2( \pm 5)$ & 0.02 \\
\hline CPP $<70$ mm Hg (\%) & $26( \pm 20)$ & $32( \pm 19)$ & 0.05 & $14( \pm 14)$ & $19( \pm 17)$ & 0.05 & $8( \pm 9)$ & $15( \pm 17)$ & 0.001 \\
\hline CPP $<80 \mathrm{~mm} \mathrm{Hg}(\%)$ & $59( \pm 24)$ & $66( \pm 21)$ & 0.04 & $41( \pm 27)$ & $48( \pm 26)$ & 0.06 & $27( \pm 21)$ & $40( \pm 27)$ & 0.001 \\
\hline CPP $<90$ mm Hg (\%) & $84( \pm 15)$ & $87( \pm 14)$ & 0.08 & $69( \pm 27)$ & $75( \pm 23)$ & 0.09 & $55( \pm 27)$ & $69( \pm 25)$ & 0.001 \\
\hline$\triangle$ CPPopt $<-10$ mm Hg (\%) & $25( \pm 14)$ & $25( \pm 13)$ & 0.94 & $18( \pm 12)$ & $20( \pm 12)$ & 0.44 & $18( \pm 11)$ & $20( \pm 11)$ & 0.19 \\
\hline$\triangle$ CPPopt \pm 10 mm Hg (\%) & $55( \pm 12)$ & $56( \pm 11)$ & 0.67 & $56( \pm 11)$ & $54( \pm 10)$ & 0.20 & $53( \pm 12)$ & $54( \pm 11)$ & 0.43 \\
\hline$\triangle$ CPPopt > 10 mm Hg (\%) & $16( \pm 9)$ & $15( \pm 9)$ & 0.42 & $21( \pm 12)$ & $21( \pm 13)$ & 0.66 & $25( \pm 15)$ & $21( \pm 12)$ & 0.08 \\
\hline PRx (coefficient) & $0.13( \pm 0.12)$ & $0.15( \pm 0.13)$ & 0.18 & $0.14( \pm 0.16)$ & $0.17( \pm 0.15)$ & 0.18 & $0.15( \pm 0.12)$ & $0.22( \pm 0.16)$ & 0.001 \\
\hline$P R x>0.05(\%)$ & $59( \pm 15)$ & $61( \pm 15)$ & 0.42 & $60( \pm 19)$ & $62( \pm 16)$ & 0.38 & $61( \pm 14)$ & $67( \pm 17)$ & 0.01 \\
\hline Body temperature $>38 \mathrm{C}(\%)$ & $15( \pm 19)$ & $13( \pm 16)$ & 0.44 & $55( \pm 29)$ & $54( \pm 27)$ & 0.93 & $51( \pm 29)$ & $61( \pm 28)$ & 0.01 \\
\hline
\end{tabular}

$p$ Values in bold and bold italics are considered statistically significant

$25 \mathrm{~mm} \mathrm{Hg} 1 \pm 1$ vs. $2 \pm 2, p$ value $<0.001$ ) (Table 2). Those with favorable outcome also had significantly lower GMT (\%) of ICP insults above $20 \mathrm{~mm} \mathrm{Hg}$ in the early and late vasospasm phases (Table 2).

The incidence of CPP below 60, 70, 80, and $90 \mathrm{~mm}$ $\mathrm{Hg}$ was higher in the early phase post-ictus and gradually decreased (Fig. 4 and Table 2). Patients with favorable outcome had significantly lower GMT (\%) of $\mathrm{CPP}<80 \mathrm{~mm} \mathrm{Hg}(59 \pm 24$ vs. $66 \pm 21, p$ value $<0.05)$, but the other fixed CPP thresholds did not differ in the early phase (Fig. 4 and Table 2). There was no association between favorable outcome and GMT (\%) of the fixed CPP thresholds in the early vasospasm phase, but favorable outcome was very significantly associated with lower GMT (\%) below all CPP thresholds (from 60 to $90 \mathrm{~mm}$ $\mathrm{Hg}$ ) in the late vasospasm phase (Table 2). There was no association between clinical outcome and $\triangle \mathrm{CPPopt}$ thresholds in any phase (Fig. 5 and Table 2) and this held true even after excluding those patients with DC surgery (data not presented). 
$\mathrm{CPP}<60 \mathrm{~mm} \mathrm{Hg}(\% \mathrm{GMT})$

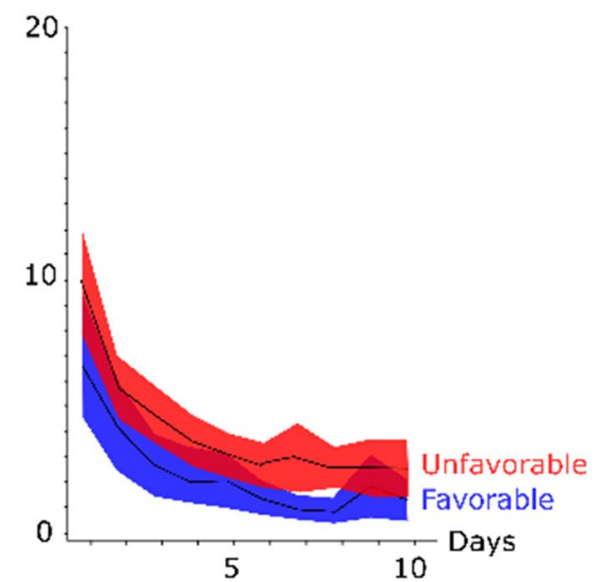

$\mathrm{CPP}<70 \mathrm{~mm} \mathrm{Hg}(\% \mathrm{GMT})$

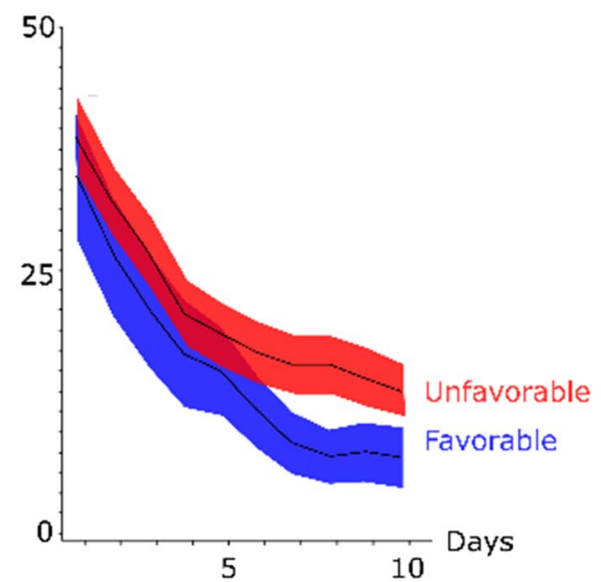

CPP $<90 \mathrm{~mm} \mathrm{Hg}(\% \mathrm{GMT})$

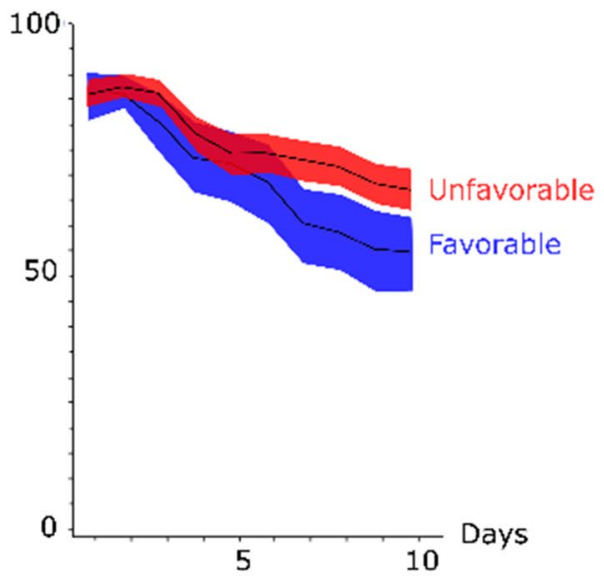

Fig. 4 Fixed cerebral perfusion pressure thresholds in relation to clinical outcome following aneurysmal subarachnoid hemorrhage-a temporal analysis the first 10 days. The trendlines indicate mean values and the shaded areas (red and blue) the 95\% confidence interval. CPP $=C e r e b r a l$ perfusion pressure and GMT $=$ Good monitoring time

Both outcome groups had a mean PRx above 0 in the early phase and both vasospasm phases (Fig. 1 and Table 2) and it was only significantly lower for those with favorable outcome in the late vasospasm phase $(0.15 \pm 0.12$ vs. $0.22 \pm 0.16, p$ value $<0.001)$. Exclusion of those who were operated with DC did not affect this association (data not presented). Both outcome groups also had similar incidence of systemic hyperthermia insults in the first two phases and those with favorable outcome had significantly lower incidence in the late vasospasm phase $(51 \pm 29$ vs. $61 \pm 28, p$ value $<0.01)$ (Table 2).

\section{Multiple Logistic Regression of Favorable Outcome for the Three Phases}

Multiple logistic regression for favorable outcome was performed for each of the three phases including the secondary insult variables that were significant in the univariate analyses in addition to age, WFNS grade, and Fisher grade as explanatory variables (Table 3). Younger age, lower WFNS grade, and lower Fisher grade were significantly associated with favorable outcome in all three phases. In the early phase, lower GMT (\%) with ICP above $20 \mathrm{~mm} \mathrm{Hg}$ was also significantly associated with favorable outcome (AUROC $=0.793$ ), as was lower GMT (\%) with ICP above $25 \mathrm{~mm} \mathrm{Hg}$ (AUROC = 0.792) in a separate outcome regression (not shown). In the early vasospasm phase, the incidence of secondary ICP and 
$\triangle$ CPPopt $<-10 \mathrm{~mm} \mathrm{Hg}(\% \mathrm{GMT})$

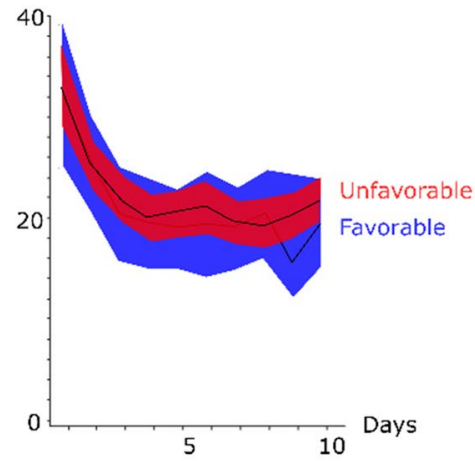

$\triangle \mathrm{CPPopt} \pm 10 \mathrm{~mm} \mathrm{Hg}(\% \mathrm{GMT})$

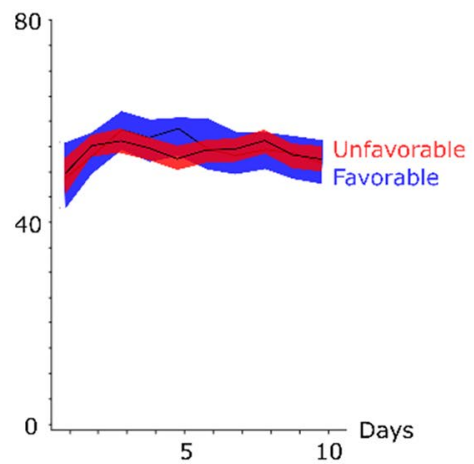

$\triangle$ CPPopt $>10 \mathrm{~mm} \mathrm{Hg}(\% \mathrm{GMT})$

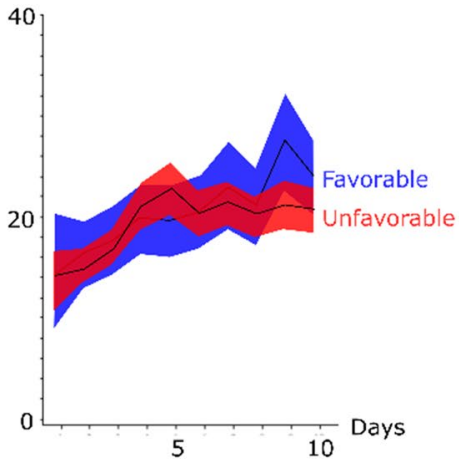

Fig. 5 Autoregulatory cerebral perfusion pressure thresholds in relation to clinical outcome following aneurysmal subarachnoid hemorrhage-a temporal analysis the first 10 days. The trendlines indicate mean values and the shaded areas (red and blue) the 95\% confidence interval. CPP $=$ Cerebral perfusion pressure, CPPopt $=$ optimal CPP (the CPP with the concurrently best autoregulation), $\triangle \mathrm{CPPopt}=\mathrm{CPP}-\mathrm{CPPopt}$, and GMT $=\mathrm{Good}$ monitoring time

Table 3 The relation between secondary insults and clinical outcome in three phases following aneurysmal subarachnoid hemorrhage - multiple logistic regression of favorable outcome

\begin{tabular}{|c|c|c|}
\hline \multirow[t]{2}{*}{ Variables } & \multicolumn{2}{|l|}{ Ictal phase } \\
\hline & Odds ratio $(95 \% \mathrm{Cl})$ & $p$ Value \\
\hline Age & $0.92(0.89-0.95)$ & 0.001 \\
\hline WFNS grade & $0.77(0.59-0.99)$ & 0.05 \\
\hline Fisher grade & $0.54(0.31-0.95)$ & 0.03 \\
\hline $\mathrm{ICP}>20 \mathrm{~mm} \mathrm{Hg}$ & $0.83(0.74-0.93)$ & 0.001 \\
\hline $\mathrm{CPP}<80 \mathrm{~mm} \mathrm{Hg}$ & $1.0(0.98-1.0)$ & 0.49 \\
\hline Variables & $\begin{array}{l}\text { Early vasospasm phase } \\
\text { Odds ratio }(95 \% \mathrm{Cl})\end{array}$ & $p$ Value \\
\hline Age & $0.94(0.91-0.97)$ & 0.001 \\
\hline WFNS grade & $0.71(0.55-0.91)$ & 0.01 \\
\hline Fisher grade & $0.39(0.22-0.70)$ & 0.002 \\
\hline $\mathrm{ICP}>20 \mathrm{~mm} \mathrm{Hg}$ & $0.95(0.89-1.01)$ & 0.07 \\
\hline $\mathrm{CPP}<60 \mathrm{~mm} \mathrm{Hg}$ & $0.96(0.87-1.07)$ & 0.49 \\
\hline Variables & $\begin{array}{l}\text { Late vasospasm phase } \\
\text { Odds ratio }(95 \% \mathrm{Cl})\end{array}$ & $p$ Value \\
\hline Age & $0.94(0.91-0.97)$ & 0.001 \\
\hline WFNS grade & $0.72(0.53-0.96)$ & 0.03 \\
\hline Fisher grade & $0.37(0.19-0.72)$ & 0.003 \\
\hline $\mathrm{ICP}>20 \mathrm{~mm} \mathrm{Hg}$ & $0.92(0.82-1.04)$ & 0.17 \\
\hline$C P P<90 \mathrm{~mm} \mathrm{Hg}$ & $0.99(0.97-0.99)$ & 0.04 \\
\hline$T>38^{\circ} \mathrm{C}$ & $0.99(0.98-1.00)$ & 0.19 \\
\hline $\mathrm{PR}_{x}$ & $0.02(0.00-0.25)$ & 0.003 \\
\hline
\end{tabular}

$p$ Values in bold italics are considered statistically significant

All three outcome regressions included age, WFNS grade, and Fisher grade as baseline variables. The secondary insult variables that were significant in the univariate analyses in each phase were analyzed. When several thresholds of, e.g., ICP (20 and $25 \mathrm{~mm} \mathrm{Hg})$ in the early phase were associated with clinical outcome, both thresholds were analyzed in separate regressions and the regression with the highest area under receiver operating curve was presented in the current table 
CPP insults were not independent predictors of favorable outcome, but a lower incidence of CPP below $90 \mathrm{~mm} \mathrm{Hg}$ in the late phase was associated with favorable outcome. The other CPP thresholds in the same phase were not independent predictors when analyzed separately. Lower mean PRx in the late phase was independently associated with favorable clinical outcome $($ AUROC $=0.807)$, as was lower GMT (\%) PRx $>0.05($ AUROC $=0.803)$ in a separate regression (not shown). However, ICP $>20 \mathrm{~mm}$ $\mathrm{Hg}$ and body temperature $>38^{\circ} \mathrm{C}$ were not independent outcome predictors in that phase.

\section{Discussion}

In the current study of 242 patients with severe aSAH that required ICP monitoring, the incidence of secondary insults and their relation to clinical outcome were related to different temporal phases post-ictus. In the early phase, ICP insults above $20 \mathrm{~mm} \mathrm{Hg}$ were more frequent and independently associated with unfavorable outcome. In the late vasospasm phase, lower CPP levels (even regarding as high thresholds as $80-90 \mathrm{~mm}$ $\mathrm{Hg}$ ) were associated with unfavorable outcome. Pressure autoregulation was disturbed in the majority of patients throughout the temporal course and most evidently so in the late vasospasm phase for those with unfavorable outcome. Deviations of absolute CPP from the PRx-derived CPPopt target were not associated with unfavorable outcome. Our findings indicate that more aggressive ICP treatment in the early phase and that higher CPP above our traditional threshold at $60 \mathrm{~mm} \mathrm{Hg}$ in the vasospasm phase could be beneficial.

\section{Intracranial Pressure in Aneurysmal Subarachnoid Hemorrhage-Thresholds and Timing of Insults}

ICP rises rapidly following intracranial aneurysm rupture and may cause an intracranial circulatory arrest. ICP may remain elevated in the early course due to global cerebral edema, acute hydrocephalus, and intracranial hemorrhage volume and in the later phase following complications to aneurysm treatment and DCI. Several previous studies have reported a relatively high incidence of ICP above 20 or $25 \mathrm{~mm} \mathrm{Hg}$ [4, 7-9], but the relation between high ICP and clinical outcome is not fully elucidated. We have previously reported that higher GMT (\%) of ICP above $25 \mathrm{~mm} \mathrm{Hg}$ was associated with clinical deterioration in aSAH, but not with long-term clinical outcome [4]. Others have reported an association between ICP above $20 \mathrm{~mm} \mathrm{Hg}$ and mortality [7], but not as an independent predictor of unfavorable clinical outcome $[7,8]$. In the current study, we evaluated the temporal dynamics of ICP insults and found that they were more frequent in the early phase post-ictus and independently predicted unfavorable outcome. This association to clinical outcome could be confounded by the detrimental etiologies for increased ICP, as mentioned above, but it held true even after adjustment for the neurological status at admission (WFNS) and structural injury (Fisher grade). This finding suggests that ICP above $20 \mathrm{~mm} \mathrm{Hg}$ is dangerous and should be avoided.

Regarding specific ICP thresholds, we found that higher GMT (\%) of both ICP above $20 \mathrm{~mm} \mathrm{Hg}$ and $25 \mathrm{~mm} \mathrm{Hg}$, respectively, was strong predictor of unfavorable outcome. This supports $20 \mathrm{~mm} \mathrm{Hg}$ as a reasonable ICP threshold after aSAH, consistent with the management protocol at our NIC and many other sites $[7,8]$. It also supports that similar ICP thresholds can be used in both aSAH and TBI [27]. However, it is possible that although ICP above $20 \mathrm{~mm} \mathrm{Hg}$ might dichotomize between acceptable and dangerously elevated ICP, lower ICP targets might be beneficial to increase CPP during vasospasm/DCI.

\section{Pressure Autoregulation and Cerebral Perfusion Pressure in Aneurysmal Subarachnoid Hemorrhage-Fixed and Autoregulatory Thresholds}

Cerebral pressure autoregulation is commonly disturbed after aSAH with development of large-vessel cerebral vasospasm that peaks between days 4 to 10 after ictus [5]. Several studies have used TCD-derived measures of vasospasm, but this method is user-dependent and does not allow for continuous measurements [11]. PRx is a well-established method for continuous evaluation of pressure autoregulation in TBI [12-14], however, its role in aSAH is less established. Previous studies on PRx in aSAH demonstrate that higher values indicative of disturbed pressure autoregulation are common and associated with lower $\mathrm{CBF}$ [16], but its relation to the risk of developing DCI is controversial [16, 22]. The association between PRx and clinical outcome is also not clear. Gaasch et al. found that PRx was significantly higher in the early phase post-ictus for patients that later developed DCI and had unfavorable outcome [22], but others have found no association between PRx and clinical outcome [20, 21]. However, the earlier publications are based on small patient populations $(<100)$ and this is to our knowledge the largest study on PRx in aSAH including 242 patients. We found that PRx was commonly above zero throughout the temporal course, indicating disturbed pressure autoregulation. PRx was also significantly higher in the late vasospasm phase for those with unfavorable outcome, but not earlier and not worse for those who developed DCI and required triple- $\mathrm{H}$ therapy. It is possible that the deterioration in PRx during the late vasospasm phase reflects a larger burden of silent DCI in these patients that explains a worse clinical outcome. However, as PRx varies with CPP [15], it could also be 
confounded by that patients with favorable outcome also had higher CPP.

In addition to being a risk factor for poor outcome and possibly DCI, PRx can be used for estimation of optimal CPP (CPPopt), i.e., the CPP that gives the lowest PRx, that can be used to individualize CPP targets [13-15, 18, 19]. Several retrospective studies in TBI have indicated that keeping the absolute CPP close to the CPPopt target could improve clinical outcome [13-15] and CPPopt management is currently being evaluated in a randomized controlled trial (RCT) in TBI (COGiTATE) [28]. Deviation from CPPopt has also been associated with worse outcome in aSAH in a smaller study including 29 patients [19], but it has not been validated in larger patient cohorts or compared with fixed CPP targets.

In the current study, CPPopt was higher for those with favorable outcome. This is paradoxical, since higher CPPopt would indicate a need to counteract an increased cerebrovascular tone such as in cases with cerebral vasospasm, whereas vasospasm is usually associated with worse clinical outcome. Future studies are needed to further explore this somehow surprising association. However, we found that there was no association between deviations of absolute CPP from CPPopt and clinical outcome in any phase post-ictus. Nevertheless, we did find that higher absolute CPP was generally associated with more favorable outcome, specifically in the vasospasm phase. The etiology for higher CPP in the vasospasm phase was rather explained by higher MAP than lower ICP (Fig. 1). The lower CPP threshold in our management protocol is $60 \mathrm{~mm} \mathrm{Hg}$, but our results suggest that even higher values above $90 \mathrm{~mm} \mathrm{Hg}$ could be beneficial in the late vasospasm phase. These findings are consistent with Schmidt et al., who found that the risk of detrimental brain tissue hypoxia and energy metabolic disturbances is relatively high even when CPP is between 60 and $70 \mathrm{~mm} \mathrm{Hg}$, but decreases above $70 \mathrm{~mm} \mathrm{Hg}$ [10].

Our results highlight that although several management principles including CPP thresholds are extrapolated from severe TBI to aSAH, the relation between CPP and clinical outcome differs. In TBI, we have previously found that CPP between 60 and $70 \mathrm{~mm} \mathrm{Hg}$ is associated with favorable outcome and CPP above $70 \mathrm{~mm} \mathrm{Hg}$ is associated with worse clinical outcome [13, 29], whereas we found that higher CPP above $70 \mathrm{~mm} \mathrm{Hg}$ was better in our aSAH patients in the current study. CPPopt targets have shown promising results in TBI [13-15], but not in the current study on aSAH. Fixed CPP targets that are slightly increased during the vasospasm phase might suit the majority of aSAH patients with a relatively homogenous pathophysiology, whereas individualized autoregulatory CPPopt targets might be more appropriate in TBI victims with a more heterogeneous pathophysiology. The lack of correlation between CPPopt targets and clinical outcome in aSAH patients could also be explained by that $\mathrm{PRx}$ and CPPopt might have a lower signal-to-noise ratio in aSAH patients that generally are treated with open EVDs. However, we and others have demonstrated that the validity of PRx and hence CPPopt is preserved when the EVD is opened [30,31]. Another explanation is that $\mathrm{PRx}$ is a global measure of pressure autoregulation, but vasospasm and DCI may be focal events [32]. Focal differences in the cerebrovascular function could even each other out when evaluated with a global measure, which could reduce the sensitivity of such events for PRx.

Although our findings indicate that higher CPP is better in the vasospasm phase, this does not necessarily support the use of vasopressors, since our patients were treated with vasopressors only if CPP was below $60 \mathrm{~mm}$ $\mathrm{Hg}$, but not to higher levels. The findings may, however, at least indicate that spontaneously high blood pressure levels (CPP levels) within reasonable limits should not be lowered. We did not analyze the explanatory variables for differences in absolute CPP levels, but is likely that patients with comorbidities, aSAH-induced cardiac dysfunction, and systemic vasodilation from hyperthermia generally had lower MAP and CPP. Future prospective trials are needed to address if higher CPP targets in the vasospasm phase could improve clinical outcome after aSAH and what treatments that should be initiated to increase CPP, e.g., including more aggressive ICP reduction with lower EVD opening pressure and MAP-augmentation by fluids and vasopressors. It is also likely that optimal MAP management depends on the concurrent condition as mentioned above.

\section{Methodological Strengths and Limitations}

The study included a uniquely, large patient population with high-resolution physiological data. We used a strict inclusion criteria so that all patients had ICP/ CPP data on all of the first 10 days, for better evaluation of the temporal dynamics in physiological variables. Hence, our patient population mainly reflects those with the most severe aSAH that required such extensive ICP monitoring, since the EVD may not be needed or can usually be discontinued earlier in more benign cases. This limits the external validity of our findings to those with the most severe aSAH. This also explains why the clinical outcome was generally poor in our study population (only $26 \%$ with favorable outcome). Furthermore, this was a retrospective, observational study and it is likely that many confounding variables could explain the relation between physiological variables and clinical outcome. We addressed this to some extent by multiple regression analyses, but we cannot exclude that other unadjusted variables 
still confounded the results. There are also some concerns about the validity of PRx and CPPopt after DC. Recent findings in TBI suggest that these measures are still valid after DC [33] and we found no impact on the results including $\mathrm{PRx}$ and CPPopt after excluding the patients with DC surgery. In addition, CPPopt might be relatively high in case of cerebral vasospasm and we cannot exclude that we did not reach high enough CPP values that fully explored the upper limit of the CPPopt curve. It is possible that a more aggressive CPP management toward higher values would have better revealed a truer CPPopt and that keeping the absolute CPP close to such values would have better discriminated between favorable and unfavorable outcome. It is also possible that the absolute PRx values were too high in general for CPPopt curves to occur.

\section{Conclusions}

The incidence of secondary insults including intracranial pressure above $20 \mathrm{~mm} \mathrm{Hg}$ and cerebral perfusion insults below 60-70 mm Hg was higher for patients with unfavorable clinical outcome after aneurysmal subarachnoid hemorrhage. The former were particularly common in the early phase. The latter were less common in the vasospasm phase, but still correlated more strongly with worse clinical outcome then when the pressure autoregulatory status was worse. Our findings indicate that more aggressive intracranial pressure treatment in the early phase and a cerebral perfusion pressure target above $70 \mathrm{~mm} \mathrm{Hg}$ in the vasospasm phase could be beneficial. Particularly, this suggests that antihypertensive agents should generally be avoided in the vasospasm phase, but it is possible that more aggressive intracranial pressure lowering treatments and/or the use of certain vasopressors could be favorable. There was no relation between deviations from CPPopt and clinical outcome, although we cannot exclude an association discernible in a larger patient population and with a different CPP management.

\section{Acknowledgements}

We express our gratitude to the personnel at the neurointensive-care unit, Uppsala University Hospital, for meticulous patient care.

\footnotetext{
Author contributions

The manuscript complies with all instructions to authors. The authorship requirements have been met and the final manuscript was approved by all authors. TSW developed the project, collected the data, and wrote the manuscript. TH and ERE developed the project, collected the data, and edited the manuscript. AL developed the project and edited the manuscript. PE developed the project, edited the manuscript, and contributed to supervision.
}

\section{Source of Support}

Open access funding provided by Uppsala University.
Ethical Approval

All procedures performed in the studies involving humans were in accordance with the ethical standards of the national research committee and with the 1964 Helsinki declaration and its later amendments. The study was approved by Uppsala University Regional Ethical Board (Dnr 2010/138 and Dnr 2010/138/1). Informed consent was obtained during neurointensive care from the next of kin.

\section{Conflict of interest}

The authors have no conflicts of interest.

\section{Open Access}

This article is licensed under a Creative Commons Attribution 4.0 International License, which permits use, sharing, adaptation, distribution and reproduction in any medium or format, as long as you give appropriate credit to the original author(s) and the source, provide a link to the Creative Commons licence, and indicate if changes were made. The images or other third party material in this article are included in the article's Creative Commons licence, unless indicated otherwise in a credit line to the material. If material is not included in the article's Creative Commons licence and your intended use is not permitted by statutory regulation or exceeds the permitted use, you will need to obtain permission directly from the copyright holder. To view a copy of this licence, visit http://creativecommons.org/licenses/by/4.0/.

\section{Publisher's Note}

Springer Nature remains neutral with regard to jurisdictional claims in published maps and institutional affiliations.

Received: 29 September 2020 Accepted: 18 November 2020 Published online: 9 January 2021

\section{References}

1. de Rooij NK, Linn FH, van der Plas JA, Algra A, Rinkel GJ. Incidence of subarachnoid haemorrhage: a systematic review with emphasis on region, age, gender and time trends. J Neurol Neurosurg Psychiatry. 2007;78(12):1365-72.

2. Diringer MN, BleckTP, Claude Hemphill J 3rd, et al. Critical care management of patients following aneurysmal subarachnoid hemorrhage: recommendations from the Neurocritical Care Society's Multidisciplinary Consensus Conference. Neurocrit Care. 2011;15(2):211-40.

3. Enblad P, Persson L. Impact on clinical outcome of secondary brain insults during the neurointensive care of patients with subarachnoid haemorrhage: a pilot study. J Neurol Neurosurg Psychiatry. 1997;62(5):512-6.

4. Ryttlefors M, Howells T, Nilsson P, Ronne-Engström E, Enblad P. Secondary insults in subarachnoid hemorrhage: occurrence and impact on outcome and clinical deterioration. Neurosurgery. 2007;61(4):704-14.

5. Connolly ES Jr, Rabinstein AA, Carhuapoma JR, et al. Guidelines for the management of aneurysmal subarachnoid hemorrhage: a guideline for healthcare professionals from the American Heart Association/American Stroke Association. Stroke. 2012;43(6):1711-37.

6. Cesarini KG, Hårdemark HG, Persson L. Improved survival after aneurysmal subarachnoid hemorrhage: review of case management during a 12-year period. J Neurosurg. 1999;90(4):664-72.

7. Zoerle T, Lombardo A, Colombo A, et al. Intracranial pressure after subarachnoid hemorrhage. Crit Care Med. 2015:43(1):168-76.

8. Heuer GG, Smith MJ, Elliott JP, Winn HR, LeRoux PD. Relationship between intracranial pressure and other clinical variables in patients with aneurysmal subarachnoid hemorrhage. J Neurosurg. 2004;101(3):408-16.

9. Magni F, Pozzi M, Rota M, Vargiolu A, Citerio G. High-resolution intracranial pressure burden and outcome in subarachnoid hemorrhage. Stroke. 2015;46(9):2464-9.

10. Schmidt JM, Ko SB, Helbok R, et al. Cerebral perfusion pressure thresholds for brain tissue hypoxia and metabolic crisis after poor-grade subarachnoid hemorrhage. Stroke. 2011;42(5):1351-6.

11. Budohoski KP, Czosnyka M, Kirkpatrick PJ, et al. Clinical relevance of cerebral autoregulation following subarachnoid haemorrhage. Nat Rev Neurol. 2013;9(3):152-63. 
12. Czosnyka M, Smielewski P, Kirkpatrick $P$, et al. Continuous assessment of the cerebral vasomotor reactivity in head injury. Neurosurgery. 1997;41(1):11-9.

13. SvedungWettervik T, Howells T, Enblad P, Lewen A. Temporal neurophysiological dynamics in traumatic brain injury: role of pressure reactivity and optimal cerebral perfusion pressure for predicting outcome. J Neurotrauma. 2019;36(11):1818-27.

14. Aries MJ, Czosnyka M, Budohoski KP, et al. Continuous determination of optimal cerebral perfusion pressure in traumatic brain injury. Crit Care Med. 2012;40(8):2456-63.

15. Steiner LA, Czosnyka M, Piechnik SK, et al. Continuous monitoring of cerebrovascular pressure reactivity allows determination of optimal cerebral perfusion pressure in patients with traumatic brain injury. Crit Care Med. 2002;30(4):733-8.

16. Johnson U, Engquist $H$, Howells $T$, et al. Bedside xenon-CT shows lower CBF in SAH patients with impaired CBF pressure autoregulation as defined by pressure reactivity Index (PRx). Neurocrit Care. 2016;25(1):47-55

17. Johnson $U$, Engquist $H$, Lewén $A$, et al. Increased risk of critical CBF levels in SAH patients with actual CPP below calculated optimal CPP. Acta Neurochir (Wien). 2017;159(6):1065-71.

18. Bijlenga P, Czosnyka M, Budohoski KP, et al. "Optimal cerebral perfusion pressure" in poor grade patients after subarachnoid hemorrhage. Neurocrit Care. 2010;13(1):17-23.

19. Rasulo FA, Girardini A, Lavinio A, et al. Are optimal cerebral perfusion pressure and cerebrovascular autoregulation related to long-term outcome in patients with aneurysmal subarachnoid hemorrhage? J Neurosurg Anesthesiol. 2012;24(1):3-8.

20. Barth M, Woitzik J, Weiss C, et al. Correlation of clinical outcome with pressure-, oxygen-, and flow-related indices of cerebrovascular reactivity in patients following aneurysmal SAH. Neurocrit Care. 2010;12(2):234-43.

21. Eide PK, Sorteberg A, Bentsen G, et al. Pressure-derived versus pressure wave amplitude-derived indices of cerebrovascular pressure reactivity in relation to early clinical state and 12-month outcome following aneurysmal subarachnoid hemorrhage. J Neurosurg. 2012;116(5):961-71.

22. Gaasch M, Schiefecker AJ, Kofler M, et al. Cerebral autoregulation in the prediction of delayed cerebral ischemia and clinical outcome in poorgrade aneurysmal subarachnoid hemorrhage patients. Crit Care Med. 2018;46(5):774-80.
23. Howells T, Elf K, Jones PA, et al. Pressure reactivity as a guide in the treatment of cerebral perfusion pressure in patients with brain trauma. J Neurosurg. 2005;102(2):311-7.

24. Wilson JL, Pettigrew LE, Teasdale GM. Structured interviews for the Glasgow outcome scale and the extended Glasgow outcome scale: guidelines for their use. J Neurotrauma. 1998;15(8):573-85.

25. Teasdale GM, Pettigrew LE, Wilson JT, Murray G, Jennett B. Analyzing outcome of treatment of severe head injury: a review and update on advancing the use of the Glasgow Outcome Scale. J Neurotrauma. 1998;15(8):587-97.

26. Sorrentino E, Diedler J, Kasprowicz M, et al. Critical thresholds for cerebrovascular reactivity after traumatic brain injury. Neurocrit Care. 2012;16(2):258-66.

27. Carney N, Totten AM, O'Reilly C, et al. Guidelines for the management of severe traumatic brain injury. Fourth Edition Neurosurgery. 2017:80(1):6-15.

28. Beqiri E, Smielewski P, Robba C, et al. Feasibility of individualised severe traumatic brain injury management using an automated assessment of optimal cerebral perfusion pressure: the COGiTATE phase II study protocol. BMJ Open. 2019;9(9):e030727.

29. Elf K, Nilsson P, Ronne-Engström E, Howells T, Enblad P. Cerebral perfusion pressure between 50 and $60 \mathrm{~mm} \mathrm{Hg}$ may be beneficial in head-injured patients: a computerized secondary insult monitoring study. Neurosurgery. 2005;56(5):962-71.

30. Aries MJ, de Jong SF, van Dijk JM, et al. Observation of autoregulation indices during ventricular CSF drainage after aneurysmal subarachnoid hemorrhage: a pilot study. Neurocrit Care. 2015;23(3):347-54

31. Howells T, Johnson U, McKelvey T, Ronne-Engström E, Enblad P. The effects of ventricular drainage on the intracranial pressure signal and the pressure reactivity index. J Clin Monit Comput. 2017;31(2):469-78.

32. Davis SM, Andrews JT, Lichtenstein $M$, et al. Correlations between cerebral arterial velocities, blood flow, and delayed ischemia after subarachnoid hemorrhage. Stroke. 1992;23(4):492-7.

33. Zeiler FA, Aries M, Cabeleira M, et al. Statistical cerebrovascular reactivity signal properties after secondary decompressive craniectomy in traumatic brain injury: a CENTER-TBI pilot analysis. J Neurotrauma 2020. 